\title{
THE COLLIMATION SYSTEM OF THE SNS TRANSFER LINES *
}

\author{
N. Catalan-Lasheras ${ }^{\dagger}$ and D. Raparia \\ Brookhaven National Laboratory, Upton, New York, 11973, USA.
}

\begin{abstract}
The High Energy Beam Transport (HEBT) and Ring to Target Beam Transport (RTBT) lines of the Spallation Neutron Source (SNS) include collimation systems that protect the line itself, as well as the systems downstream. Due to the one-pass mechanism in a transfer line, collimation can only provide protection against accidental losses while shaping the beam is reserved for multi-pass systems as rings. Efficiency has to be improved by increasing the impact parameter in the collimator in the first passage. In the HEBT line the use of stripers for collimation of the $\mathrm{H}^{-}$ beam enhances the final capture efficiency but introduces some limitations in the layout geometry. In the RTBT, protection is provided by the optics of the line and collimator optimization is reduced to considerations of length and the amount of free space available. We revisited the design of the collimation systems for both transfer lines attending to various criteria including loss scenarios and flexibility. With the revised design, we estimated the final performance and cleaning efficiency under nominal conditions for both systems.
\end{abstract}

\section{INTRODUCTION}

Because of the high availability and reliability expected from the Spallation Neutron Source (SNS), one of the main concerns when designing the accelerator is radio-activation and residual dose produced by uncontrolled beam loss [1]. When designing the accumulator ring and transfer lines of the SNS accelerator we provide special locations to capture the beam losses as collimators and beam dumps. A very large efficiency of the collimator systems is required to keep the uncontrolled beam loss limited to 1-2 Watt of beam power per meter [2]. Besides the multi-stage collimation system designed for the ring [3], the two transfer lines of the SNS (from the linac to the accumulator ring (HEBT) and from the ring to the spallation target (RTBT)), are equipped with collimation systems to keep beam losses limited to designated locations in the line. The final efficiency of these cleaning systems is defined by the first and only passage of the beam through the collimators.

The collimation systems of the HEBT line are covered in sections 2 and 3 while section 4 addresses the collimators in the RTBT line. In each section, we introduce the requirements and limitations when designing the collimation system. We describe their final layout and working principle. An evaluation of the final performance and the

\footnotetext{
* Work performed under the auspices of the U.S. Department of Energy

†catalan@bnl.gov
}

expected cleaning efficiency under nominal conditions is presented.

\section{HEBT TRANSVERSE COLLIMATION}

The rms emittance of the beam coming from the linac is expected to be small $\left(\epsilon_{r m s}=0.5 \pi \mathrm{mm} \cdot \mathrm{mrad}\right.$ unormalised $)$ but large tails in the distribution may contain a fraction of the beam larger than $10^{-4}$. With a beam power of up to 2MW and beam losses spread along few meters, the residual radiation does not satisfy the hands-on maintenance criteria. We need to provide a transverse collimation system upstream in the line to prevent losses on the achromat, cavities and injection in the ring.

The minimum aperture in the HEBT line is given by the two RF cavities used for spreading the beam momentum and correcting the central energy [4]. The cavities acceptance is $A_{x}=26 \pi \mathrm{mm} \cdot \mathrm{mrad}$. With a small sets of collimators in each plane, the main function is to prevent the transport along the line of $\mathrm{H}^{-}$ions with emittance larger than the line acceptance. Collimators provide little additional shaping capability.

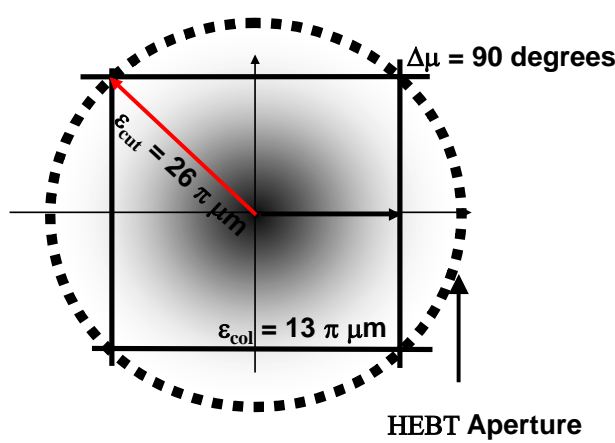

Figure 1: Cuts induced by the striper foils in normalized phase-space.

\subsection{System layout}

To remove the transverse halo from the beam, we introduce adjustable carbon foils in the path of the beam to intercept the tails. After the passage of the $H^{-}$ion through the foil, the two electrons are removed and the $\mathrm{H}^{-}$focusing lattice deviates the proton tails into large massive absorbers [5].

The aperture of the foils is given by the acceptance of the HEBT line. For transverse collimation of the halo, we have two pair of facing foils in each horizontal and vertical plane 


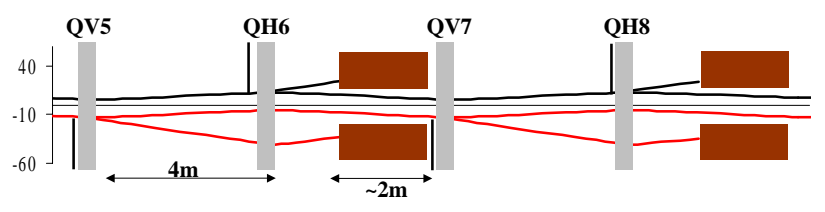

Figure 2: Layout of the HEBT collimation system and proton beam trajectories. Top half corresponds to the horizontal plane while the vertical is represented by the bottom half.

We locate them 90 degrees of phase advance apart to provide the most efficient cut of the phase space. To protect the cavities, the final emittance of any escaping particle must be less than $A_{H E B T}=26 \pi \mathrm{mm} \cdot \mathrm{mrad}$. Hence, the foils nominal aperture is set to $A_{\text {foil }}=13 \pi \mathrm{mm} \cdot \mathrm{mrad}$ (Fig. 1). The horizontal and vertical stripping foils are located at the maximum of the beta function in each plane to enhance the resolution of the system.

Once the position and acceptance of the foils is fixed, the aperture and position of the absorber is decided by the focusing lattice and the required impact parameter.

We transported a proton beam created at each foil with any emittance value in the vertical plane. The beam clears the quadrupoles aperture and hits the absorber with a large impact parameter. The minimal impact parameters on the absorbers of the proton beam produced in each of the stripers is shown in table 1.

\subsection{Estimated performance}

For $H^{-}$collimation by charge exchange the interception of the secondary halo by the absorber is $100 \%$ efficient. Still, due to small impact parameters, the protons have a finite probability of escaping the absorber without being removed.

Using the values for the impact parameter on table 1, we simulate the passage of the protons through the material of the absorber with a Monte Carlo code [6]. The absorption efficiency for the secondary $\mathrm{H}+$ beam generated at each foil is given in tabe 1 . For efficiency simulations we used a simplified model of the absorbers with a geometry presenting a equivalent mass of stainless steel. A more realistic model is used for secondary particle production and radiation calculations [7].

\begin{tabular}{|c|c|c|c|c|c|}
\hline \multirow[t]{2}{*}{ Name } & \multirow{2}{*}{$\begin{array}{l}\text { Apert. } \\
{[\mathrm{mm}]}\end{array}$} & \multicolumn{2}{|c|}{ Impact param. } & \multirow{2}{*}{$\begin{array}{l}\text { Eff. } \\
{[\%]}\end{array}$} & \multirow[b]{2}{*}{ Abs } \\
\hline & & {$[\mathrm{mm}]$} & [mrad] & & \\
\hline Scr $1 \mathrm{U} / \mathrm{D}$ & 13 & 12.3 & -5.9 & 94.3 & 1 \\
\hline Scr $1 \mathrm{~L} / \mathrm{R}$ & 17 & 4.3 & 6.7 & 91.1 & 1 \\
\hline Scr 2 U/D & 13 & 15.0 & -4.1 & 95.9 & 2 \\
\hline Scr 2 L/R & 17 & 3.3 & 6.1 & 88.7 & 2 \\
\hline
\end{tabular}

Table 1: Aperture, impact parameter and simulated efficiency for every pair foil/absorber. The initial acceptance at the foils correspond to $13 \pi \mathrm{mm} \cdot \mathrm{mrad}$. The efficiency has been calculated for $1 \mathrm{GeV}$ energy protons.
The particles escaping the collimator loss a significant fraction of their total momentum by ionization with the absorber material. They are lost along the achromat or captured by the longitudinal collimation system.

These values of efficiency are calculated for the nominal aperture of the foils. Opening or closing them produces a different impact parameter at the absorber and changes the final efficiency. In the same way, the absorption efficiency was calculated for an energy of $1 \mathrm{GeV}$. Larger energy result on slightly better efficiency and a different loss pattern downstream from the collimator.

\section{HEBT LONGITUDINAL COLLIMATION}

The tightest requirement for the longitudinal extent of the $H^{-}$beam is given by the RF capture in the ring. Previous studies of the longitudinal dynamics have concluded that any beam above $\pm 4 \mathrm{MeV}$ ends in the gap between consecutive bunches inducing intolerable losses during extraction. A less stringent limit is the momentum acceptance in the HEBT achromat where dispersion is non zero.

We thus locate a charge exchange foil in a high dispersion region in the HEBT achromat to remove the longitudinal tails of the beam before entering in the ring. The losses expected in this collimator are much larger than in the transverse case due to the tighter longitudinal acceptance. Also the incertitude about the energy jitter and momentum spread coming from the superconducting linac translate into a larger beam loss budget.

Again, because the one-pass condition in a transfer line, only particles with the right betatrin phase are intercepted by the foil and striped even if they have large momentum deviation and emittance. The adjustable foil shaves the $H^{-}$ beam halo when the following condition is fulfilled

$$
x=\sqrt{\epsilon_{x} \cdot \beta} \cdot \sin (\phi)+D \cdot \delta p / p_{0} \geq x_{c u t}
$$

where $x_{c u t}=17 \mathrm{~mm}$ is the foil horizontal position and $\mathrm{D}=6.9 \mathrm{~m}$ and $\beta=20 \mathrm{~m}$ are the dispersion and beta function at the foil.

A acceptance plot is shown in Fig. 3 where the variables are the horizontal emittance and relative momentum deviation of the $\mathrm{H}^{-}$ion. The escaping halo clears the HEBT aperture for the emittance range of interest. However, some particles escape with energy deviation above $4 \mathrm{MeV}$ which contribute to the beam in the gap inside the ring. Assuming gaussian distributions, a nominal beam coming from the linac with a transverse emittance of $\epsilon_{r m s}=0.28 \pi \mathrm{mm} \cdot \mathrm{mrad}$ and a energy spread of $\Delta T_{r m s}= \pm 0.72 \mathrm{MeV}$ will have a less than a fraction $10^{-4}$ above the limit determined by the foil. A Montecarlo integration predicts an interception efficiency of the gaussian tails only of $14 \%$. For a beam with an emittance and momentum deviation twice the nominal values area predicts an efficiency of $39 \%$.However, large momentum tails are removed more efficiently than large emittance particles. The interception efficiency for particles with momentum deviation larger than $4 \mathrm{MeV}$ is better 


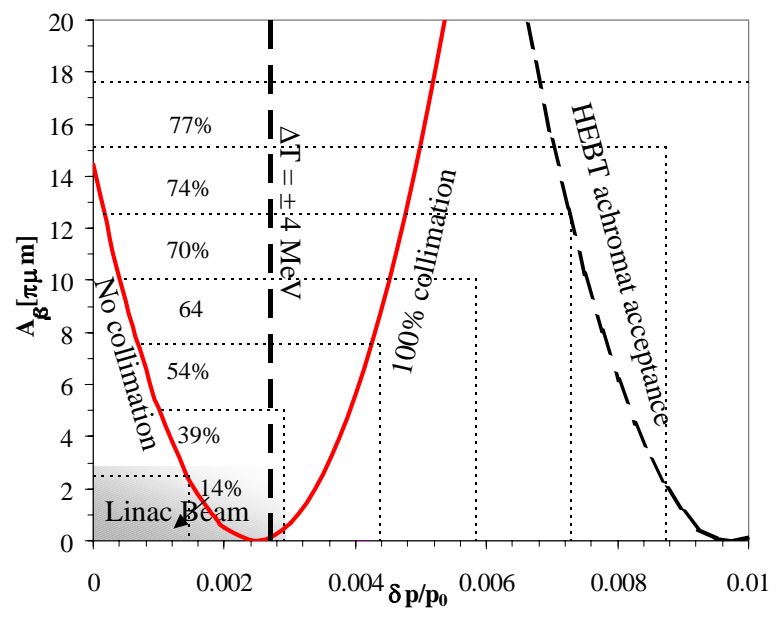

Figure 3: Collimator acceptance in terms of betatron amplitude vs. $\delta p / p_{0}$. The geometry acceptance of the HEBT achromat and the momentum spread limit at $\pm 4 \mathrm{MeV}$ are represented by dashed lines.

than $90 \%$ ensuring that the population of the gap in the ring is less than $\approx 10^{-5}$ of the total beam.

After being striped, the proton beam is deviated by the dipole field into a large absorber outside the circulating beam trajectory. The vacuum pipe geometry after the foil has been adjusted to clear the dipoles and drive the $\mathrm{H}^{+}$ beam on the absorber [8]. In this case, the absorption efficiency in the collimator is $100 \%$ as protons are deviated from the main path and hit the absorber located outside the accelerator.

\section{RTBT COLLIMATION}

\subsection{Requirements}

Unlike the HEBT line, where the beam is small and the micro bunch power is reduced, the beam circulating along the RTBT line has the full beam power and a considerable size compared to the line aperture. Besides, a full pulse transported along the line with a large closed orbit deviation may damage the target vessel. The main purpose of the RTBT collimation system is to capture the beam in the event of extraction kicker misfire. The optics and aperture have been adjusted so that, if one of the fourteen extraction kickers fails, the beam is transported through the line without scraping and the deviation on the target is under $2 \mathrm{~mm}$ (see Fig. 4). When more than one kicker fail at the same time, the beam is intercepted by two collimators. The position of the collimators has been chosen between the locations with large orbit deviation and is shown in Fig. 4 superimposed to the closed orbit deviation. The phase advance between them is $\approx 135^{\circ}$.

\subsection{Estimated performance}

Fig. 5 shows the beam center and extension at the two collimator when two extraction kickers fail simultaneously.

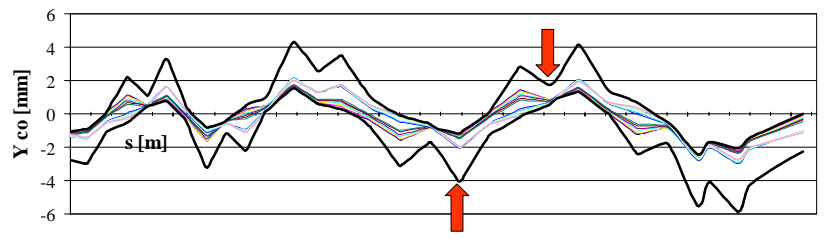

Figure 4: Vertical closed orbit deviation along the RTBT. Thin lines correspond to one kicker misfire. Bold lines are the maximum and minimum closed orbit deviation when two kickers fail.
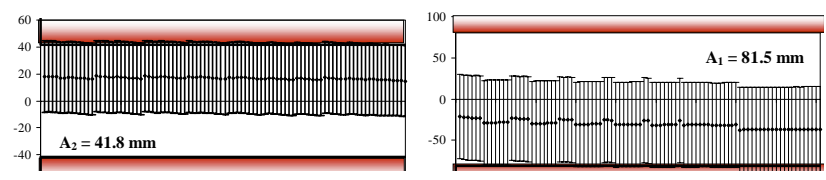

Figure 5: Beam position and size at the two RTBT absorbers after two extraction kicker missfire. The aperture of the collimators corresponds to $400 \pi \mathrm{mm} \cdot \mathrm{mrad}$.

As for the HEBT, we have simulated the absorption efficiency of the collimators using a Monte-Carlo simulation. We calculate the initial conditions entering the collimator assuming a uniform beam of $160 \mathrm{\pi mm} \cdot \mathrm{mrad}$ total emittance. The average impact parameter and angle in the absorbers are $3.4 \mathrm{~mm}$ and $-4.8 \mathrm{mrad}$ in the first absorber and $1.6 \mathrm{~mm}$ and $3.7 \mathrm{mrad}$ in the second, leading to an average absorption efficiency of $99.8 \%$ and $83.5 \%$, respectively.

\section{CONCLUSIONS}

We have defined the requirements of the collimation systems in the transfer lines of the SNS accelerator. The fact that the beam only makes one passage through the cleaning system makes capture more difficult than in multi-pass systems and further tightens the requirements. Protection is the major concern to design the system leaving the shaping of the beam for the collimation system in the ring.

\section{REFERENCES}

[1] J. Wei et al. Phys. Rev. ST Accel. Beams 3, 080101 (2000)

[2] W. Chou and N. Mokhov (Ed.), The 7th ICFA MiniWorkshop on Beam Halo and Scraping, Lake Como,WI September 13-15, 1999

[3] N. Catalan-Lasheras et al. Phys. Rev. ST Accel. Beams 4, 010101 (2001)

[4] D. Raparia et al., " the NSNS High Energy Beam Transport Line” PAC 1997, Vancouver

[5] H. Ludewig et al., "Collimator Systems for the SNS Ring". PAC 1999, New York, 1999

[6] J.B. Jeanneret and T. Trenkler "K2: A Software Package Evaluating Collimation Systems in Circular Colliders". CERN SL 94-105 AP.

[7] H. Ludewig et al., These proceedings

[8] P. He. These proceedings 